\author{
Man-Un Ung ${ }^{1}$ \\ Benzhuo Lu, ${ }^{1,2}$ \\ J. A. McCammon ${ }^{1,2,3,4}$ \\ ${ }^{1}$ Department of Chemistry \\ and Biochemistry, \\ University of California at \\ San Diego, La Jolla, \\ CA 92093-0365, USA \\ ${ }^{2}$ Center for Theoretical \\ Biological Physics, \\ University of California at \\ San Diego, La Jolla, \\ CA 92093-0365, USA \\ ${ }^{3}$ Department of Pharmacology, \\ University of California at \\ San Diego, La Jolla, \\ CA 92093-0365, USA \\ ${ }^{4}$ Howard Hughes Medical \\ Institute, University of \\ California at San Diego, \\ La Jolla, CA 92093-0365, USA

\section{E230Q Mutation of the Catalytic Subunit of cAMP-Dependent Protein Kinase Affects Local Structure and the Binding of Peptide Inhibitor} \\ Received 14 September 2005 ; \\ revised 6 December 2005 ; \\ accepted 8 December 2005 \\ Published online 19 December 2005 in Wiley InterScience (www.interscience.wiley.com). DOI 10.1002/bip.20434
}

\begin{abstract}
The active site of the mammalian cAMP-dependent protein kinase catalytic subunit (C-subunit) has a cluster of nonconserved acidic residues - Glu127, Glu170, Glu203, Glu230, and Asp241that are crucial for substrate recognition and binding. Studies have shown that the Glu230 to Gln mutant (E230Q) of the enzyme has physical properties similar to the wild-type enzyme and has decreased affinity for a short peptide substrate, Kemptide. However, recent experiments intended to crystallize ternary complex of the E230Q mutant with MgATP and protein kinase inhibitor (PKI) could only obtain crystals of the apo-enzyme of E230Q mutant. To deduce the possible mechanism that prevented ternary complex formation, we used the relaxed-complex method (Lin, J.-H., et al. J Am Chem Soc 2002, 24, 5632-5633) to study PKI binding to the E230Q mutant C-subunit. In the E230Q mutant, we observed local structural changes of the peptide binding site that correlated closely to the reduced PKI affinity. The structural changes occurred in the F-to-G helix loop and appeared to hinder PKI binding. Reduced electrostatic potential repulsion among Asp241 from the helix loop section and the other acidic residues in the peptide binding site appear to be responsible for the structural change. (C) 2005 Wiley Periodicals, Inc. Biopolymers 81: 428-439, 2006

This article was originally published online as an accepted preprint. The "Published Online" date corresponds to the preprint version. You can request a copy of the preprint by emailing the Biopolymerseditorial office at biopolymers@wiley.com
\end{abstract}

edu

Correspondence to: Benzhuo Lu; e-mail: blu@mccammon.ucsd.

Biopolymers, Vol. 81, 428-439 (2006)

(C) 2005 Wiley Periodicals, Inc. 
Keywords: C-subunit of cAMP-dependent protein kinase; E230Q; local structural change; PKI binding; docking

\section{INTRODUCTION}

cAMP-dependent protein kinase (protein kinase A, PKA) is one of the smallest and simplest protein kinases. ${ }^{1}$ Its crystal structure was the first of a protein kinase to be solved ${ }^{2}$ and often serves as the prototype of the protein kinase family because of its conserved catalytic core. ${ }^{3}$ The catalytic subunit (C-subunit) of PKA is comprised of two lobes - a small lobe that has a nucleotide-binding domain and a large lobe that has a peptide binding site. ${ }^{2}$ The peptide binding site in the large lobe recognizes a wide variety of peptide substrates with the phosphorylation site ( $\mathrm{P}$ site, nomenclature adopted from Knighton et al. ${ }^{2}$ ) preceded by two basic residues, typically arginines at P-2 and $\mathrm{P}-3$ positions of the consensus site, and a large hydrophobic $(\mathrm{P}+1)$ residue following the $\mathrm{P}$ site. ${ }^{4}$

A cluster of acidic residues (Glu127, Glu170, Glu203, Glu230, and Asp241) in the C-subunit were identified as being among the binding determinants of peptide substrates by charged-to-alanine mutagenesis. ${ }^{5}$ Except Asp241, these acidic residues were responsible for binding the arginines on the consensus sequence of the substrate peptides. They are not conserved throughout the protein kinase family and are found only in PKA C-subunit. ${ }^{6}$ Glu230 is positioned in the center of the peptide binding site and is approximately $15 \AA$ from the ATP-binding site. It recognizes the P-2 Arg of the peptide substrates and contributes significantly to the electrostatic properties of the peptide binding site. ${ }^{7}$ Mutation of Glu230 to Gln (E230Q) demonstrated the importance of this residue. The E230Q mutant enzyme has reduced negative electrostatic potential on the surface of the peptide binding site. ${ }^{7}$ The mutant is also defective in binding peptide substrates and deficient in catalyzing the phosphoryl transfer reaction. ${ }^{8}$ Kinetic data showed that the E230Q mutation affected the binding of ATP with only 2 -fold perturbation but induced a 200 -fold reduction in the binding of the peptide substrate, Kemptide. $^{8}$

Another important feature of the E230Q mutant is the loss of the synergistic binding of MgATP and protein kinase inhibitor $\operatorname{PKI}(5-24)$ to the mutant. The mutant was unable to form ternary complex crystals in the presence of MgATP and PKI. ${ }^{9}$ However, studies on the E230Q apo-enzyme crystal structure, ${ }^{9}$ thermostability, ${ }^{8,10}$ and hydrogen-deuterium exchange ${ }^{10}$ suggest that the structure of the E230Q mutant is very similar to the wild-type C-subunit. Hence, it is very likely that the altered surface electrostatic profile of the E230Q mutant causes the observed defective kinetic properties of the enzyme. . $^{7,10}$

We applied the relaxed-complex method described by Lin et al. ${ }^{11}$ to study the effects of the E230Q mutation on PKI binding. We obtained a qualitative estimation of the probability of PKI docking to the Csubunits. We found that, in the E230Q mutant, there are fewer best-docked PKI conformations compared to the wild-type and that an altered PKI binding mode is found to be associated with an altered F-to-G helix loop conformation. This local conformational change occurs between Pro237 and Pro243 of the loop, a section that packs closely against a peptide inhibitor in the crystal structure of the ternary complex. The distance between the $\alpha$-carbon atoms of Glu170 and Asp241, which measures the width of the peptide binding site, decreased significantly during molecular dynamics (MD) simulation of the mutant. The altered peptide binding site may sterically hinder PKI binding, affecting the affinity of PKI for the E230Q mutant. Estimates of the electrostatic potential energy associated with the nonconserved acidic residues in the peptide binding site indicate that the E230Q mutant has a weaker negative electrostatic potential in the site compared to the wild-type. Altered electrostatic potential energy is also observed to be associated with the altered F-to-G helix loop conformation.

\section{METHODS AND MATERIALS}

\section{System Setup and Simulation Method}

Two crystal structures of PKA C-subunit were used to initiate these studies. The crystal structure of the ternary complex of the recombinant mouse form of the wild-type PKA C-subunit with MnATP and PKI bound ${ }^{12}$ (PDB ID: 1ATP) was determined at $2.2 \AA$ resolution. The recombinant mouse form of the C-subunit apo-enzyme with the Glu230to-Gln mutation ${ }^{9}$ (PDB ID: $1 \mathrm{SYK}$ ) was determined at $2.8 \AA$ resolution. The charges of the two phosphorylated residues (Thr197 and Ser338) in the C-subunit crystal structures were obtained by using Gaussian ${ }^{13}\left(6-31+\mathrm{G}^{*}\right.$ basis set) together with the RESP module in the AMBER ${ }^{14}$ version 7.0 package. The parameters of the missing side-chains and hydrogens were filled in using the parm 99 parameter set of 
AMBER 7.0. In the setting up of the MD simulation, the Csubunit was immersed in a $\left(78 \times 94 \times 76 \AA^{3}\right)$ water box and neutralized by addition of $\mathrm{Cl}^{-}$counterions using the AMBER Leap module; the whole simulated system consisted of 45,238 atoms. The electrostatic interactions were calculated with the Particle Mesh Ewald method, ${ }^{15}$ in which a $9.0 \AA$ cut-off radius was used for the direct interactions calculation. SHAKE algorithm ${ }^{16}$ was used to constrain all bond lengths involving hydrogens. The system was first relaxed by 25,000 steps of conjugate-gradient energy minimization and then coupled to a heat bath heating from 0 to $300 \mathrm{~K}$ within $20 \mathrm{ps}$. The system was equilibrated at $300 \mathrm{~K}$ for $100 \mathrm{ps}$, followed by 10,000 ps of production run to generate a trajectory for structural fluctuation analysis. Snapshots of trajectory were saved every $1 \mathrm{ps}$.

From the MD simulations of the wild-type and the E230Q mutant C-subunits, snapshots of the enzymes were taken at 50-ps intervals from 1000 to 6000 ps. Thus, 100 dynamic snapshots were generated for each simulation. Since no known conservative water molecule is crucial to PKI docking, ${ }^{17}$ all water molecules were omitted from the snapshots. Using the relatively rigid backbone of the large lobe, ${ }^{18}$ these dynamic snapshots were superimposed onto the large lobe of 1ATP from residue 125 to 232 and from residue 255 to 310 .

\section{AutoDock Simulations}

Partial charges, polar hydrogens, and solvation parameters were added to the dynamic snapshots in preparation for computing AutoDock 3.0 ${ }^{19}$ grid maps. Nonpolar hydrogens were merged with the carbon atom they were attached to and the united-atom charge parameters were added to the snapshots using the parameters provided by AMBER. The charges of the polar hydrogens were provided by AMBER. The charges of the two phosphorylated residues were added as described above. Solvation parameters were added to the snapshots using the AutoDock 3.0 force field. ${ }^{20}$ Atomic affinity grid maps and an electrostatic grid map were calculated for each atom type in the ligand for each protein snapshot using AutoGrid 3.0. An $\left(80 \times 90 \times 100 \AA^{3}\right)$ rectangular grid map with $0.375 \AA$ spacing between grid points was centered on the peptide binding site of $\mathrm{C}$-subunit for docking simulations.

The original coordinates of the ligand, $\operatorname{PKI}(5-24)$, were obtained from the 1ATP coordinate file. United-atom charges and other parameters were prepared the same as described above. It is known that when free PKI binds to the peptide binding site of the C-subunit, the disordered PKI undergoes considerable conformational changes and becomes ordered. ${ }^{21}$ Hence the backbone and all sidechains, except that of the P-2 Arg, of the PKI were set rigid. We also recognize the limited number of flexible bonds allowed for a ligand in AutoDock 3.0. The bonds between CA:CB, CB:CG, CG:CD, and CD:NE of P-2 Arg were set as flexible.

In the automated docking simulations, we used the default setting suggested by the program with some varia- tions. Each docking trial started with 100 randomly generated populations of binding orientations and would go through 750,000 energy evaluations. The maximum number of generations was set at 27,000 and only 1 top individual would survive in each generation. 50 trials of docking were done for each snapshot.

AutoDock 3.0 has been used successfully as a tool to model interactions between proteins and small ligands. ${ }^{22-25}$ However, PKI was substantially larger than the ligands used in the mentioned experiments. Furthermore, the dynamic snapshots of the C-subunit had high flexibility. The docked PKI therefore had relatively large fluctuations compared with the crystallographic PKI in 1ATP. The scoring scheme used in AutoDock may therefore not be sufficient to indicate the correct binding mode. Hence, in order to select the best-docked PKI binding mode with the regular, crystallographic PKI conformation, we used the following criteria, in addition to the AutoDock scoring scheme, for selecting the best-docked PKI conformation among the top five clusters of the docked results: (1) the displacement of the docked PKI backbone atoms, Thr5:CA, Asp9:CA, Arg15:CA, Arg19:CA, and His23:CA were all less than 3.0 $\AA$ away from their corresponding locations in the crystallographic PKI and (2) the distance between the docked P-2 Arg side-chain and the Glu230 or Gln230 side chain, measured from the guanidino carbon atom, P-2 Arg:CZ, to the $\gamma$ carboxyl carbon atom, Glu230:CD or Gln230:CD, is less than $4.5 \AA$.

To test whether PKI was suitable as a ligand in the docking simulation, we used 1ATP, a crystallographic C-subunit ternary complex with closed conformation, as a testing scaffold. The crystallographic PKI in the ternary complex was removed and various parameters described above were added to the protein. We performed a 100-run trial docking simulation of PKI. The trial docking simulation gave 100 best-docked PKI conformations under the AutoDock scoring scheme and the two additional criteria suggested above. The evaluation gave one single cluster of PKI binding modes that matched the crystallographic PKI binding mode with root mean square deviation (RMSD) ranging from 0.37 to $0.52 \AA$. The result indicates that, although the ligand used in the docking simulation was relatively large, AutoDock 3.0 was able to predict the correct binding mode of PKI with high accuracy.

\section{Electrostatics Calculations}

The electrostatic field was calculated by solving the Poisson-Boltzmann equation with $\mathrm{APBS}^{26}$ version 0.3.2. The AMBER charge and atomic radius parameters were used. Both the coarse grid dimensions and the fine grid dimensions were set at $\left(90 \times 90 \times 90 \AA^{3}\right)$, and the number of grid points was set at 161 in each dimension. The dielectric constant of the solvent was set at 80 and that of the protein interior was set at 2.0. The ionic strength was equivalent to $0.145 M$ sodium chloride. ${ }^{7}$ The radii of ions were $2.0 \AA$, while the solvent radius was $1.4 \AA$. The system temperature was set at $298.15 \mathrm{~K}$. 

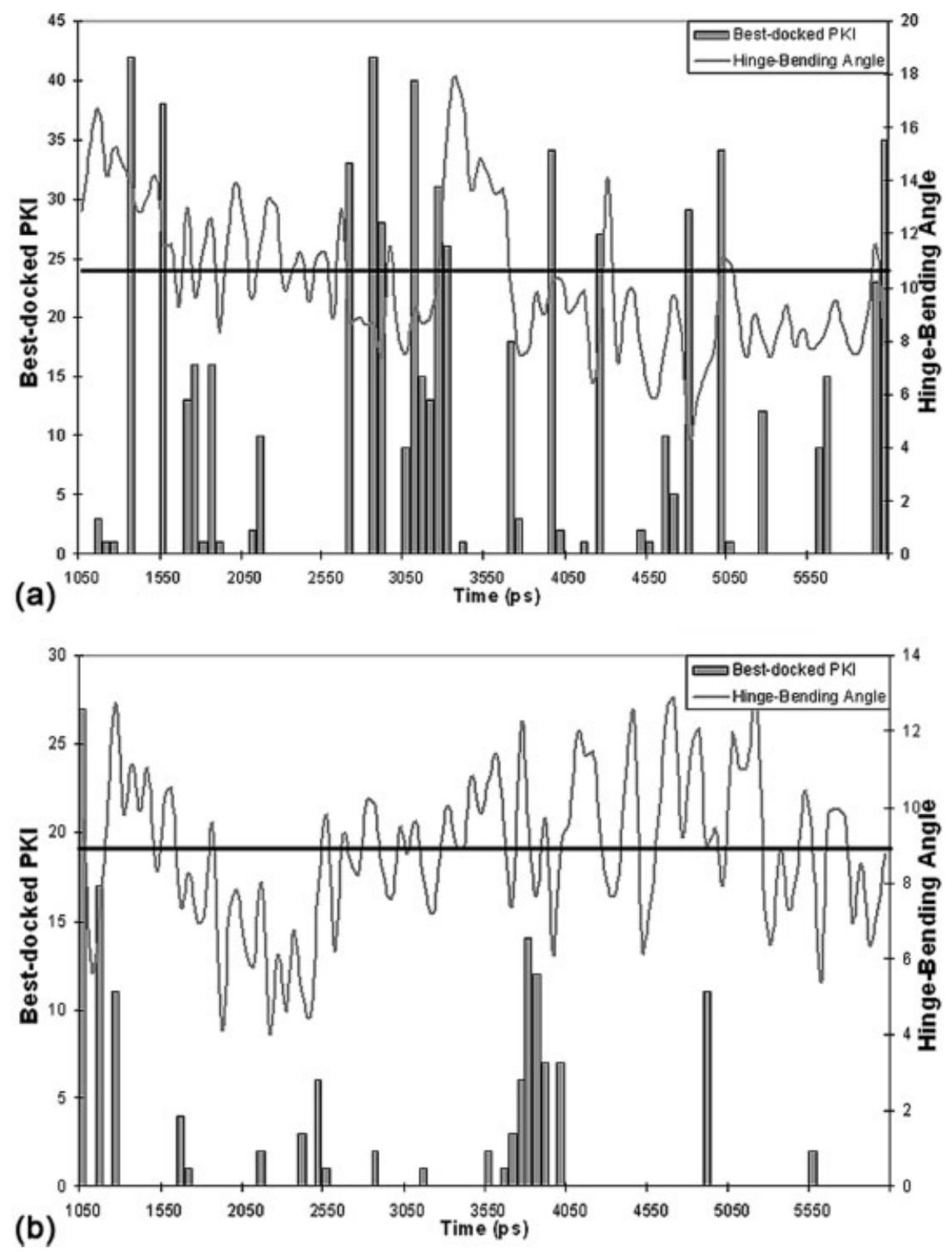

FIGURE 1 Comparison of the number of the best-docked PKI conformations and the hingebending angle between the small and the large lobes of (a) the wild-type and (b) the E230Q mutant $\mathrm{C}$-subunits. The solid black line represents the median hinge-bending angle of the snapshots.

To estimate the strength of the electrostatic interactions in the peptide binding site, the electrostatic potential energy arising from Glu170, Glu203, Glu230/Gln230, and Asp241 of the C-subunit peptide binding site was calculated using the approximation

$$
V_{\mathrm{E}}=\sum_{i j} \frac{q_{i} q_{j}}{4 \pi \varepsilon(r) r} \quad \varepsilon(r)=r
$$

where $V_{\mathrm{E}}$ is the electrostatic potential energy, $q$ is the atomic charge, $\varepsilon(r)$ is the distance-dependent solvent dielectric constant, and $r$ is the distance between two atoms. All atoms of these residues were used in the calculation and their charges were provided by AMBER. The interior electrostatic interaction in a residue was not calculated in this experiment, therefore, in the summation in Eq. (1), $i$ and $j$ belong to different residues.

\section{RESULTS AND DISCUSSION}

\section{Protein Dynamics of the C-Subunits}

The small and large lobes of the PKA C-subunit undergo hinge-bending motion in solution. This hinge-bending motion of the $\mathrm{C}$-subunit might affect the binding of PKI to the peptide binding site on the large lobe ${ }^{27}$ due to long-range intramolecular communication. Hence, we wanted to determine the effect of the hinge-bending motion on PKI docking. 
To quantify the hinge-bending motion between the small and large lobes of the C-subunit, a method we used before ${ }^{28}$ was adopted to calculate the hingebending angle between the lobes. Figure 1 depicts the relationship between the hinge-bending motion of the lobes and the number of best-docked PKI conformations for the wild-type and the E230Q mutant. The median hinge-bending angle of the wild-type was about $9.6^{\circ}$ with RMSD $2.9^{\circ}$ and that of the E230Q mutant was about $8.9^{\circ}$ with RMSD $2.0^{\circ}$. In contrast, the angle of 1 ATP with a closed conformation was measured to be $4.0^{\circ}$. Therefore, all the snapshots of the wild-type and the E230Q mutant adopted open conformations during the MD simulations. The varying angle during the simulations shows that the enzyme underwent dynamic fluctuation while maintaining an open conformation; the hinge-bending motion observed is consistent to the C-subunit openclosed motion observed by Tsigelny et al. ${ }^{29}$

Summing up the number of PKI dockings with the best-docked binding mode (Figure 2a) from the dynamic snapshots, we found that the wild-type Csubunit had more PKI dockings than the E230Q mutant, which parallels the experimental results. ${ }^{7,8,10}$ In Figure 1a, the best-docked PKIs of the wild-type scattered throughout the whole spectrum of the MD simulation. Dockings appeared both at large hinge-bending angles between the lobes $\left(>12^{\circ}\right)$, e.g., during 1250-1550, 1900-2150, and 3350-3600 ps, and at small hinge-bending angles $\left(\sim 8^{\circ}\right)$, e.g., during 2700-3200, 4750-4900, and 5100-6000 ps. In Figure 1b, the best-docked PKIs of the E230Q mutant scattered between 1050 and 4150 ps, but not beyond. Similar to the wild-type, the best-docked PKIs of E230Q mutant associated with both large hinge-bending angles (e.g., 2550-4100 ps) and small hingebending angles (e.g., 1800-2500 ps). The results from Figure $1 \mathrm{a}$ and $1 \mathrm{~b}$ suggest that the dynamic fluctuation of the open conformation does not significantly affect PKI docking to the wild-type and the E230Q mutant C-subunits.

We also adopted two other methods that describe the open-closed conformation of the C-subunit: Glu170-Tyr330 distance that measures the openness of the C-terminal "gate",30 and Ser53-Gly186 distance that measures the openness of the glycine-rich loop. ${ }^{31}$ The median of these two distances measured in the dynamic snapshots of the wild-type and the E230Q mutant (data not shown) are comparable to the corresponding distances in the open conformation crystal structure of $1 \mathrm{CTP} .{ }^{32,33}$ When these two distances are compared to the number of best-docked PKI conformations in the corresponding snapshots, we do not see obvious correlation between the fluctu- ation of the distances and the number of best-docked PKIs. These results are consistent with the finding using the hinge-bending angle, suggesting that PKI docking is not much affected by the dynamic fluctua-
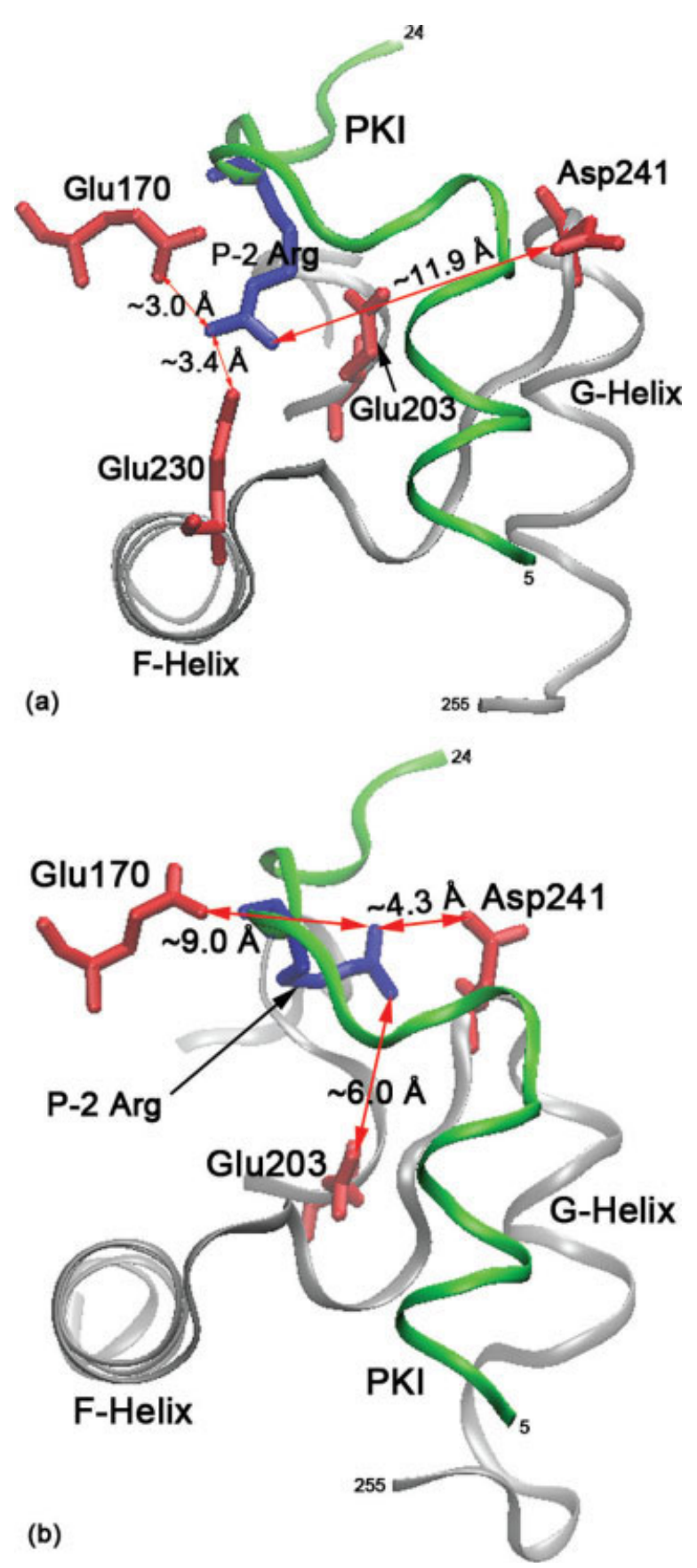

FIGURE 2 P-2 Arg of PKI interacts directly with (a) Glu170 and Glu230 in 1ATP, (b) Asp241 in the second period of E230Q mutant MD simulation. The peptide binding site of the C-subunit is shown in silver and the bound PKI in green. Acidic residues Glu170, Glu203, Glu230, and Asp241 are rendered in red and the basic residue of PKI, P-2 Arg, is shown in blue. 
tion of the open conformation. Since fewer successful dockings are found for the mutant than for the wildtype, and in neither case do we find a correlation with the degree of openness of the hinge, we therefore examined more localized interactions.

\section{Local Structural Change in E230Q Affects PKI Binding Mode}

In the docking simulations of the E230Q mutant C-subunit, a significant number of PKI molecules assumed a binding mode that did not appear in the wild-type (Figure 2a). This altered PKI binding mode (Figure 2b) appears between 4200 and 6000 ps of the MD simulation. The most striking feature of this altered PKI binding mode is that P-2 Arg of the PKI twists toward and interacts with Asp241 of the C-subunit. In the regular, crystallographic PKI binding mode (Figure 2a), the typical distance between P-2 Arg:NH1 and Asp241:OD1 was $~ 12.0 \AA$; the distance between P-2 Arg:NH1 and Glu170:OE1 was $\sim 3.0 \AA$; the distance between P-2 Arg: NH2 and Glu203:OE1 was $~ 4.5 \AA$; and the distance between P-2 Arg:NH1 and Glu230:OE1 was 3.5 ̊. P-2 Arg interacts strongly with Glu170, Glu203, and Glu230 but weakly with Asp241. In contrast, the distance between P-2 Arg and Asp241 in the altered PKI binding mode was less than $4.5 \AA$ in general, making this a strong electrostatic interacting pair. Furthermore, the distance between P-2 Arg and Glu170 was $~ 9.0$ $\AA$; the distance between P-2 Arg and Glu203 was $\sim 6.0 \AA$; and the distance between P-2 Arg and Gln230 was $\sim 10.5 \AA$. Thus, these pairs only have weak to moderate electrostatic interactions in the altered PKI binding mode. The E230Q mutant thus has a drastic change in the local electrostatic interaction network. Among these changes, the P-2 Arg:Asp241 interacting pair has a particularly dramatic alteration. Hence, Asp241, which is located in the F-to-G helix loop of the C-subunit, might have a critical role in disrupting the binding of PKI to the E230Q mutant.

\section{Distances among the Acidic Residues in the Peptide Binding Site}

To demonstrate that the location of Asp241 might affect PKI docking, the position of Asp241 was measured for all snapshots. Glu170 was used as a reference residue. The backbone $\alpha$-carbon atoms coordinates of Glu170 and Asp241 were used to calculate the distance between the two residues. This distance tracks the distance between the C-helix and the F-to$\mathrm{G}$ helix loop; it can also be considered as an indicator of the width of the peptide binding site. The measured distance was compared to the number of best-docked PKI molecules in each snapshot (Figure 3). The crystallographic Glu170-Asp241 distance in 1ATP was $18.5 \AA$. The median Glu170-Asp241 distance of the wild-type C-subunit snapshots was $20.2 \AA$ with RMSD $0.9 \AA$ (Figure 3a). The Glu170-Asp241 distance of the wild-type C-subunit did not have significant fluctuation during the MD simulation. The bestdocked PKIs scattered evenly throughout the spectrum and had binding modes similar to the regular binding mode (Figure 2a). This implies that the peptide binding site has the optimal conformation for PKI docking when the Glu170-Asp241 distance is close to $19.0 \AA$.

For the E230Q mutant (Figure 3b), there were two distinct medians for the Glu170-Asp241 distance. The median distance in the first MD simulation period (1050 to 4150 ps) was $19.4 \AA$ with RMSD $0.7 \AA$ The Glu170-Asp241 distance in this period was close to the crystallographic distance in 1ATP; the peptide binding site might assume an optimal peptide docking conformation. For these conformations, PKI is able to dock to the E230Q mutant with a binding mode similar to the regular PKI binding mode, though in fewer numbers compared to the number of best-docked PKIs in the wild-type. During the second MD simulation period (4200 to $6000 \mathrm{ps}$ ), the median dropped from 19.4 to $14.8 \AA$ with RMSD $0.61 \AA$. The $4.6 \AA$ decline in the median Glu170-Asp241 distance indicates significant change in the width of the peptide binding site. This MD simulation period of the mutant has virtually no PKI docking with the regular PKI binding mode, but instead shows the altered PKI binding mode (Figure $2 b$ ). The reduced width of the peptide binding site might have a significant role in causing the inability of the E230Q mutant to bind PKI with the regular binding mode.

In addition to the change in the Glu170-Asp241 distance seen in the second MD simulation period of the E230Q mutant, there are other considerable changes in the distances among Glu170, Glu203, Gln230, and Asp241, all measured with the $\alpha$-carbon atom coordinates (Table I). These distances correspond to the distances between the $\mathrm{P}+1$ loop and the F-to-G helix loop (Glu203-Asp241); the F-helix and the F-to-G helix loop (Glu/Gln230-Asp241); the Chelix and the P+1 loop (Glu170-Glu203); the C-helix and the F-helix (Glu170-Glu/Gln230); and the $\mathrm{P}+1$ loop and the F-helix (Glu203-Glu/Gln230). Except for the Glu170-Gln230 distance, they have considerable change between 4150 and 4200 ps. Markedly, the distances involving Asp241 have the most significant change: Glu170-Asp241 distance decreased by $\sim 4.6 \AA$, Glu203-Asp241 distance decreased 

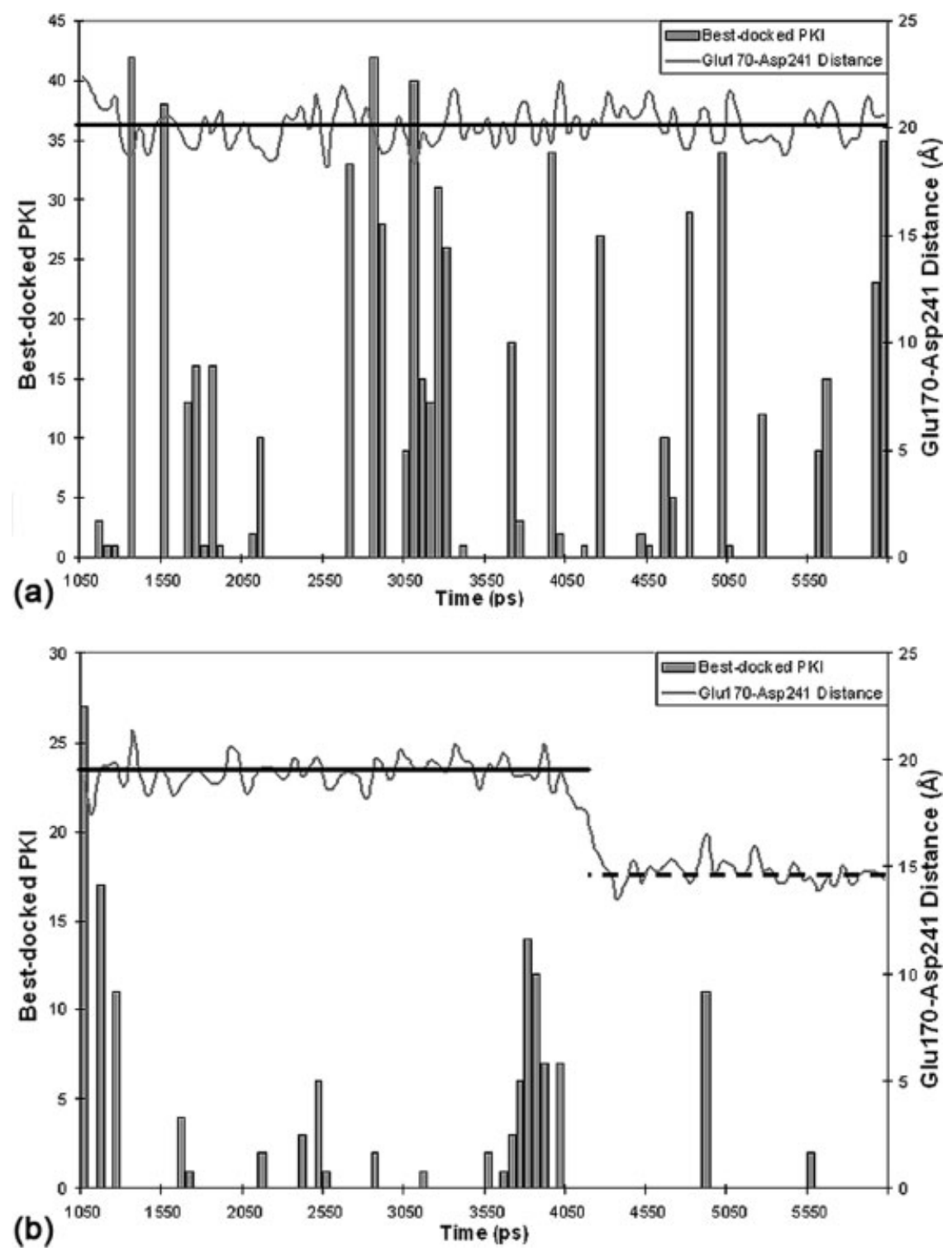

FIGURE 3 Comparison of the number of the best-docked PKI conformations and the distance between Glu170 and Asp241 in (a) the wild-type and (b) the E230Q mutant C-subunits. The solid black straight line represents the median of the Glu170-Asp241 distance [(a) about $20.2 \AA$; (b) about $19.4 \AA$ ]; dotted line in b represents the median of the distance in the second MD simulation period (about $14.8 \AA$ ). Starting from 4200 ps, the median distance dropped about $4.6 \AA$ in the second simulation period.

by $\sim 2.5 \AA$, and Gln230-Asp241 distance decreased by $\sim 3.0 \AA$.

\section{F-to-G Helix Loop Conformational Change}

Since Asp241 is part of the F-to-G helix loop, we believe that the F-to-G helix loop might be involved in causing the reduced number of best-docked PKI conformations. The section of the loop (Phe238 to Gln242) that is flanked by the two proline residues,
Pro237 and Pro243, is of particular interest. This section extends from Phe238 to Gln242 and includes Asp241. As shown in the work of Lu et al., ${ }^{28}$ this loop section had high RMSD and was highly flexible in solution. The entire section is exposed to the solution and has long-range electrostatic interactions among its Asp241 and Glu170 from the C-helix $(\sim 19.0 \AA)$, Glu203 from the $\mathrm{P}+1$ loop $(\sim 11.0 \AA)$, and Glu230 from the F-helix $(\sim 15.0 \AA)$ measured with their $\alpha$ carbon atom coordinates. It also packs closely against a section of PKI from Phe10 to Arg15, which corre- 
Table I Median Acidic Residue Distances ${ }^{\mathrm{a}}$ in 1ATP, Snapshots of the Wild-type and the E230Q Mutant MD Runs

\begin{tabular}{|c|c|c|c|c|c|}
\hline \multirow[b]{2}{*}{ Interacting Pair } & \multicolumn{5}{|c|}{ Distance $(\AA)$} \\
\hline & 1ATP & $\begin{array}{c}\text { Wild-type } \\
(1050-6000 \mathrm{ps})\end{array}$ & $\begin{array}{c}\text { E230Q } \\
(1050-4150 \mathrm{ps})\end{array}$ & $\begin{array}{c}\text { E230Q } \\
(4200-6000 \mathrm{ps})\end{array}$ & $\begin{array}{c}\text { E230Q Distance } \\
\text { Changed }\end{array}$ \\
\hline Glu170-Asp241 & 18.5 & 20.1 & 19.4 & 14.8 & -4.6 \\
\hline Glu203-Asp241 & 11.0 & 11.0 & 10.9 & 8.4 & -2.5 \\
\hline Glu/Gln230 & 14.3 & 15.6 & 15.4 & 12.4 & -3.0 \\
\hline Glu170-Glu203 & 12.2 & 13.5 & 13.5 & 14.5 & +1.0 \\
\hline Glu170-Glu/Gln230 & 9.2 & 8.5 & 8.6 & 8.4 & -0.2 \\
\hline Glu203-Glu/Gln230 & 8.2 & 9.2 & 9.0 & 10.0 & +1.0 \\
\hline
\end{tabular}

${ }^{a}$ The distance was calculated between the $\mathrm{CA}$ atom of the residues.

${ }^{\mathrm{b}}$ Glu230 in 1ATP and wild-type C-subunit; Gln230 in E230Q mutant C-subunit.

sponds to $(\mathrm{P}-6)$ to $(\mathrm{P}-11)$. Hence, any significant change in this section of the F-to-G helix loop might affect the binding of substrate with similar structure to $\mathrm{PKI}$ and has residues beyond $(\mathrm{P}-6)$.

In the wild-type $\mathrm{C}$-subunit and the first MD simulation period of E230Q mutant (1050 to $4150 \mathrm{ps),} \mathrm{the}$ Phe238-to-Gln242 of the F-to-G helix loop section had RMSDs ranging from 0.7 to $2.7 \AA$ and had a median of $1.6 \AA$ compared to the corresponding section in 1ATP. In contrast, we observed high RMSDs in the second MD period of the E230Q mutant (4200 to $6000 \mathrm{ps}$ ). The RMSD ranged from 2.5 to $5.3 \AA$ and had a median of $4.1 \AA$. Comparing the E230Q mutant F-to-G helix loop RMSDs to the number of bestdocked PKI conformations (see Figure 6), we found a strong correlation between the two. Most of the bestdocked PKIs appear in the first MD simulation period. Furthermore, they usually appear when the F-to$\mathrm{G}$ helix loop RMSD value is below the median value. The altered PKI binding mode was observed exclusively in the second MD simulation period and virtually no PKIs with regular binding mode appeared.

The significant difference in the F-to-G helix loop RMSD indicates that a local conformational change has occurred in this particular section of the E230Q mutant C-subunit (Figure 4a). The observed F-to-G helix loop conformational change accounts for the changes of Glu170-Asp241 distance and the F-to-G helix loop RMSD. The loop section flips up and closes in to the $\mathrm{C}$-helix and the $\mathrm{P}+1$ loop, which narrows the width of the peptide binding site. This conformational change might affect the proper packing of PKI in the peptide binding site. To achieve maximum binding surface with the altered F-to-G helix loop, the ordered PKI is likely forced to dock to the peptide binding site in a slightly different manner. This altered binding mode might affect the distances between the residues in the peptide binding site and the PKI. The overall effect might bring P-2 Arg of the PKI in proximal to Asp241, allowing them to interact effectively (Figure 2b), while moving it away from previously interacting residues.

Of all the dynamic snapshots from the 6000-ps MD simulation of the E230Q mutant C-subunit, the snapshots prior to 4150 ps had an F-to-G helix loop similar to the corresponding conformation in 1ATP, while those beyond 4200 ps had the altered conformation. The F-to-G helix loop maintained the altered conformation firmly in the extended MD simulation from 6000 to $10,000 \mathrm{ps}$. We therefore believe the E230Q mutant C-subunit maintains the altered F-to$\mathrm{G}$ helix loop conformation in the solution instead of the regular conformation that the wild-type C-subunit assumes.

\section{Electrostatic Interactions and Conformational Change}

The negative potential around the peptide binding site is mainly contributed by Glu127, Glu170, Glu203, Glu230, and Asp241. Glu170, Glu203, and Glu230 form a negatively charged "pocket" that interacts strongly with P-2 Arg of the PKI molecule, while Asp241 flanks the back of P-2 Arg and interacts weakly with it (Figure 2a).

Glu170, Glu230, and Asp241 are labeled on the isopotential surfaces in Figure 5. Glu203 is not labeled because it is buried underneath the isopotential surfaces of Glu170 and Asp241. In the wild-type (Figure 5a), Glu170, Glu203, Glu230, and Asp241 contribute to the strong negative potential in the peptide binding site. Using Eq. (1), we estimated the median electrostatic potential energy among these residues to be $+16.9 \mathrm{kcal} / \mathrm{mol}$ with RMSD $2.2 \mathrm{kcal} / \mathrm{mol}$, for all the dynamic snapshots. The strong electrostatic repulsion among Asp241 and other acidic residues 


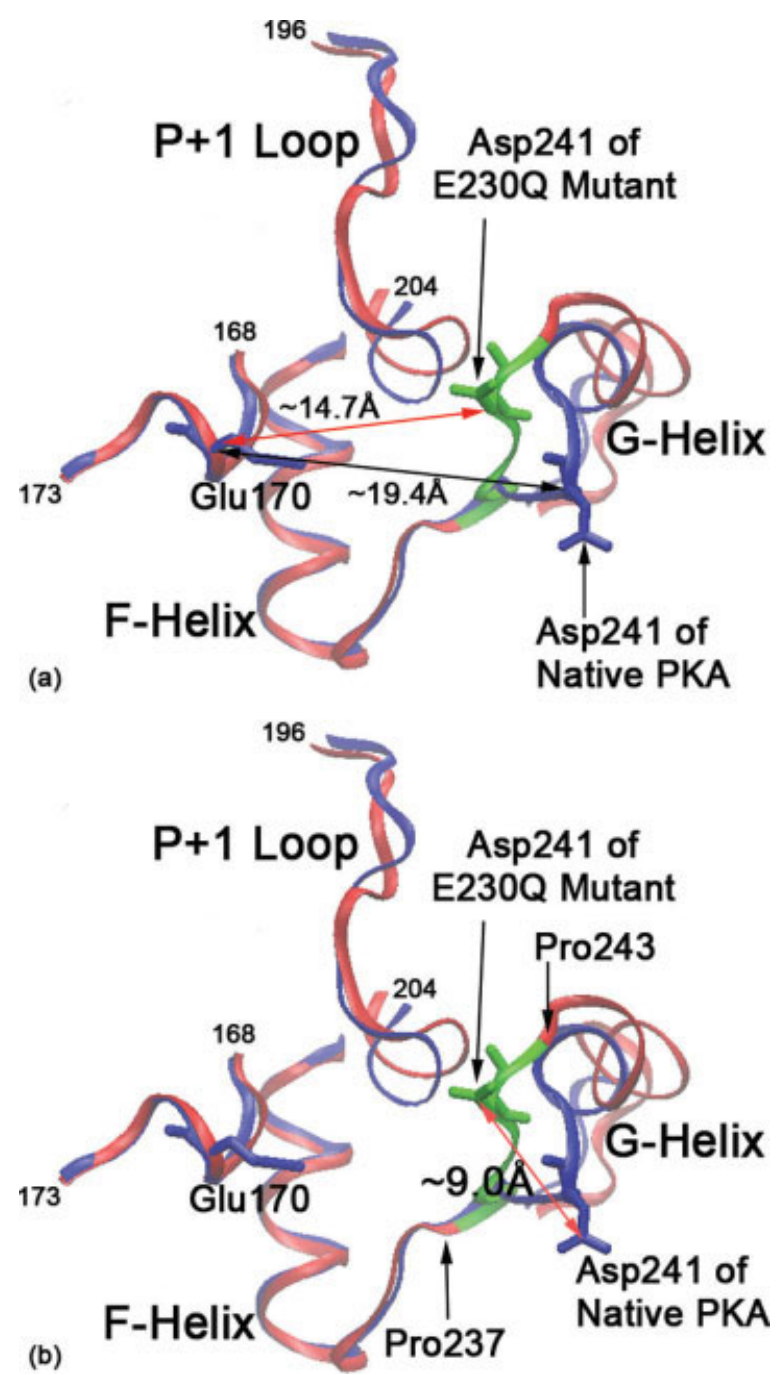

FIGURE 4 Comparison of the local structure of the peptide binding site of the wild-type and the E230Q mutant Csubunits. (a) The Glu170-Asp241 distance in the wild-type and the E230Q mutant. (b) The movement of the Asp241 carboxyl side-chain. Red ribbons represent the E230Q mutant and blue ribbons represent the wild-type. The green ribbon represents the Phe238-to-Gln242 section of the F-to-G helix loop of the E230Q mutant. In $b$, the $\beta$-carboxyl carbon atom of Asp241 was displaced from its normal position ranging from 5.3 to $10.5 \AA$, with median at $\sim 9.0 \AA$. The orientation of Asp241 $\beta$-carboxyl group had also changed from parallel to perpendicular relative to the F-helix.

(Glu170, Glu203, and Glu230) in the peptide binding site might play a role in keeping these acidic residues and their corresponding helices apart (Table I), mediating the conformation of the loop to adopt certain shape. In turn, the F-to-G helix loop, as an integral part of the peptide binding site, might influence the overall structure of the site through maintaining the binding site at an optimal width for PKI binding. Hence, the peptide binding site might be able to sense the electrostatic environment through Asp241 of the F-to-G helix loop.

In the E230Q mutant (Figure 5b), the negative electrostatic potential of the peptide binding site is observably less than that of the wild-type (Figure 5a). Using Eq. (1), we estimated the median electrostatic potential energy among Glu170, Glu203, Gln230, and Asp241 to be $+7.0 \mathrm{kcal} / \mathrm{mol}$ with RMSD 0.9 $\mathrm{kcal} / \mathrm{mol}$ for the snapshots in the first MD simulation period (1050 to $4150 \mathrm{ps}$ ), and $+8.9 \mathrm{kcal} / \mathrm{mol}$ with RMSD $1.7 \mathrm{kcal} / \mathrm{mol}$ for the snapshots in the second MD simulation period (4200 to 6000 ps). Compared to the electrostatic potential energy of the wild-type, the mutant has an 8.0 to $10.0 \mathrm{kcal} / \mathrm{mol}$ decrease in the potential energy due to the charged-to-neutral mutation of the Glu230. The mutation weakens the overall negative potential in the peptide binding site. This is in accord with the prediction made by Tsigelny et al. ${ }^{7}$ Hence, the E230Q mutant should have weaker affinity for PKI in part due to the loss of the negative potential in the peptide binding site.

We observed two distinct values of the electrostatic potential energy among Glu170, Glu203, Gln230, and Asp241 of the E230Q mutant peptide binding site (Figure 6). The first MD simulation period (1050 to $4150 \mathrm{ps}$ ) and the second MD simulation period (4200 to $6000 \mathrm{ps}$ ) have about $1.9 \mathrm{kcal} / \mathrm{mol} \mathrm{dif-}$ ference in median potential energy. The potential energy also correlates closely to the number of bestdocked PKI conformations. In the first MD simulation period, where the potential energy was about $+7.0 \mathrm{kcal} / \mathrm{mol}$, the best-docked PKIs scattered in the period. The number of best-docked PKI is less than that of the wild-type, probably due to the weakened electrostatic interaction between the E230Q mutant and the PKI. In the second MD simulation period, the potential energy was about $+8.9 \mathrm{kcal} / \mathrm{mol}$. The energy change suggests that the F-to-G helix loop adopts an altered conformation, which brings Asp241 closer to the $\mathrm{P}+1$ loop and the C-helix and slightly increases the electrostatic potential energy in the region. The slightly increased electrostatic potential energy, which correlates to the increased negative electrostatic potential strength, should increase the affinity of the E230Q mutant for PKI. However, virtually no best-docked PKI is found in this period. This suggests that steric hinderance caused by the F-to-G helix loop conformational change precludes PKI binding.

The repulsion among the acidic residues (Glu170, Glu203, and Asp241) clustered around the peptide binding site of the E230Q mutant might mediate the structure of the peptide binding site for peptide recognition; reduced repulsion might distort the electro- 


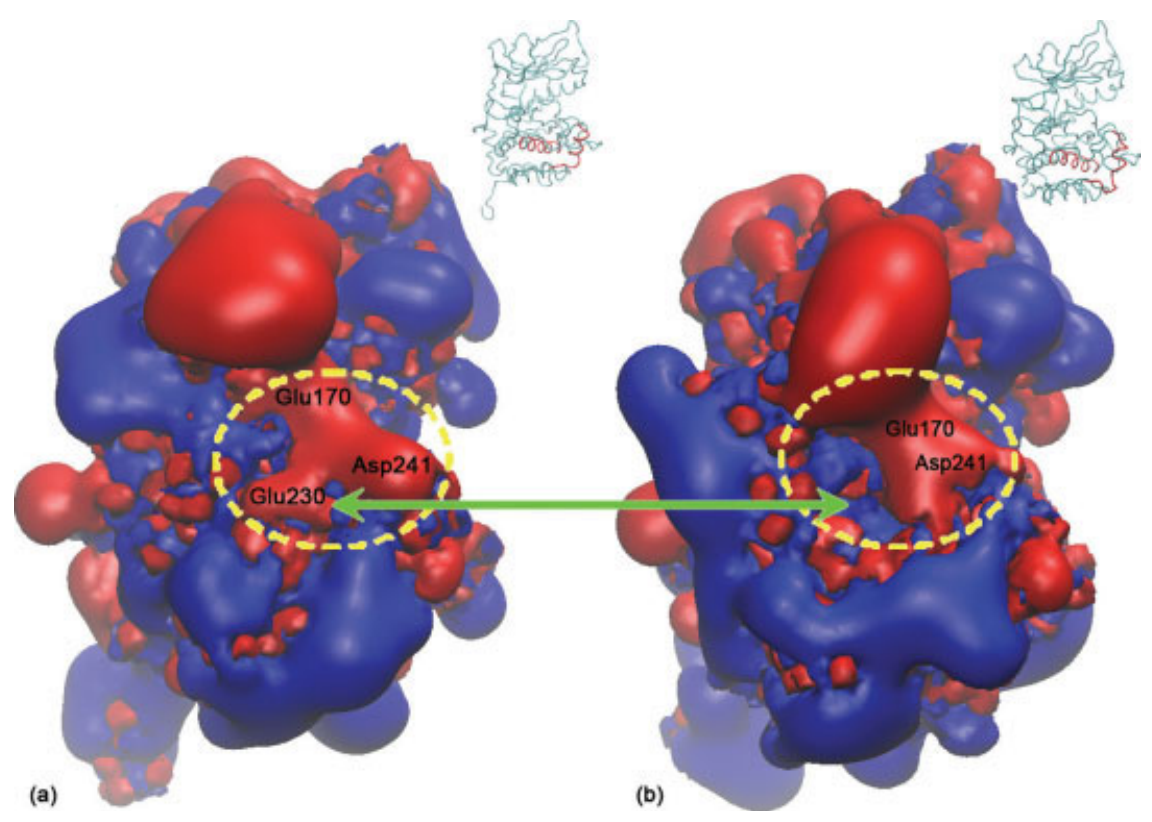

FIGURE 5 Electrostatic isopotential surfaces of (a) the wild-type (left) and (b) the E230Q mutant C-subunits (right). The green ribbon on the upper right illustrates the orientation of the molecule. The red surface corresponds to the isopotential contour $-1 k T / e$, and blue surface corresponds to the isopotential contour $+1 k T / e$, where $k$ is the Boltzmann constant, $T$ is the Kelvin temperature, and $e$ is the electronic charge. Glu170, Glu230, and Asp241 are labeled. The region that binds the consensus sequence of substrate peptides is circled with the yellow dotted line. The missing Glu230 negative electrostatic potential in b is indicated by the green two-way arrow.

static networks and result in structural change of the peptide binding site. We compared the electrostatic energy with the F-to-G helix loop RMSD (Figure 6), an indicator of the conformation of this loop. We found that the two values changed in concert during both the first and the second MD simulation periods, with sharp increases between 4150 and 4200 ps. This suggests a strong link between the electrostatic potential in the peptide binding site and the conformation of the F-to-G helix loop.

Wild-type C-subunit has strong electrostatic repulsion in the peptide binding site. This repulsion may be responsible for upholding the binding site structure by the energetic balance between the electrostatic repulsion and the other nonelectrostatic attractive interactions. The incoming positively charged P-2 Arg during PKI binding partly compensates the negative electrostatic potential among Glu170, Glu203, Glu230, and Asp241 of the peptide binding site. ${ }^{7}$ This compensation may induce the overpower of the other interactions, which may shorten the distances slightly among the loops and helices in the peptide binding site by allowing the acidic residues to come closer to each other, as evidenced by the shortened distances among these residues in 1ATP compared to the wild-type apo-enzyme (Table I). This shortening in distances may allow stron- ger electrostatic interaction between the C-subunit and the PKI while providing better packing effect. Conversely, the E230Q mutant has less negative potential, which corresponds to weaker electrostatic repulsion among the acidic residues, in the peptide binding site compared to the wild-type. This perturbation might first be tolerated (1050 to $4150 \mathrm{ps}$ ) but eventually disrupt the balance between the electrostatic repulsion and the other interactions. The peptide binding site may adopt an altered structure by the conformational change of the F-to-G helix loop, as seen in the period between 4200 and 6000 ps (Figure 4), to return to an energetic balance between the electrostatic repulsion and the other interactions. However, this structural change might abolish PKI binding as the altered loop structure hinders the packing of the (P-6)-to-(P-11) section of PKI. This interaction might severely reduce the binding affinity for PKI, making the E230Q mutant unable to form a ternary complex with PKI and MgATP.

It is worth noting that the E230Q mutant C-subunit has a 200 -fold reduction in the affinity for Kemptide. ${ }^{8}$ However, the primary factor that contributes to the reduced affinity should be the loss of P-2 Arg:Glu230 electrostatic interaction. The F-to-G helix loop should not affect Kemptide binding since the F-to-G helix loop conformational change affects primarily the resi- 


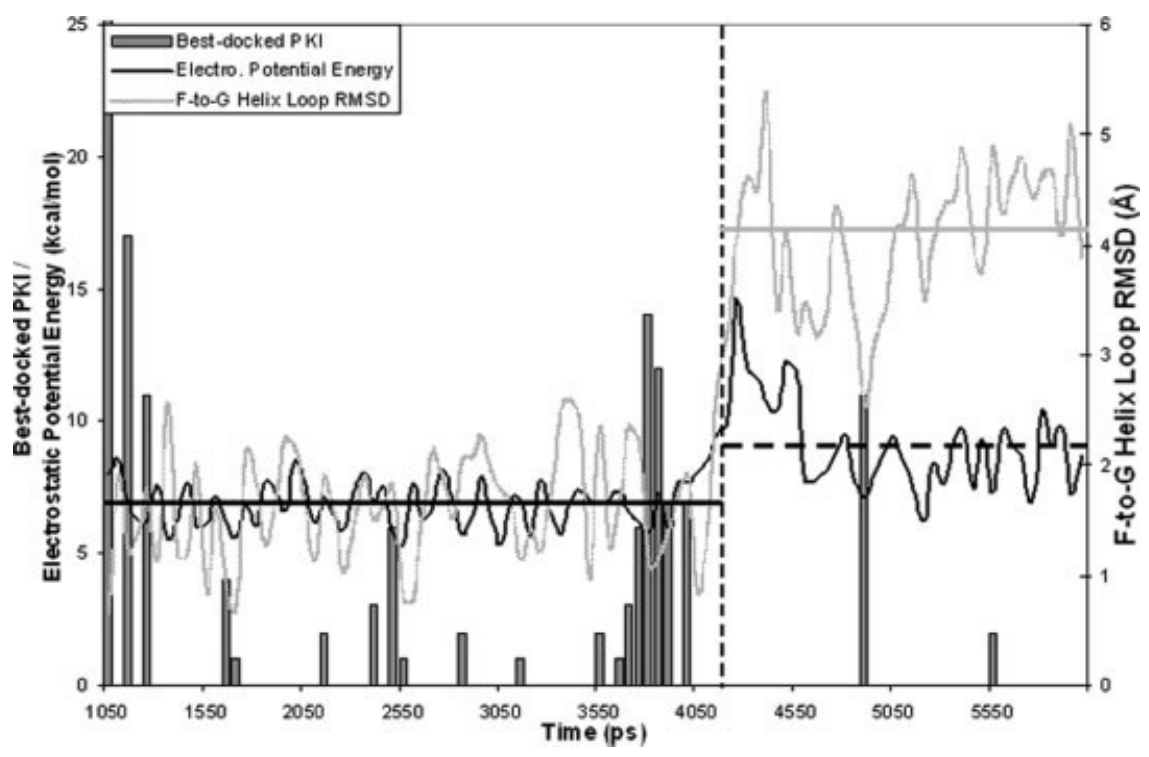

FIGURE 6 Comparison of the number of best-docked PKI conformations, F-to-G helix loop RMSD, and the electrostatic potential energy among Glu170, Glu203, Gln230, and Asp241 of the peptide binding site of the E230Q mutant C-subunit. The median F-to-G helix RMSD and the median potential energy in the first MD simulation period (about $+7.0 \mathrm{kcal} / \mathrm{mol}$ and $1.7 \AA$ ) share the same black solid straight line since the lines representing them overlap coincidently; the gray solid straight line represents the median F-to-G helix RMSD in the second MD simulation period (about $4.1 \AA$ ); and the black dotted straight line represents the median potential energy in the second MD simulation period (about $+8.9 \mathrm{kcal} / \mathrm{mol}$ ). The vertical dotted line indicates the time between 4150 and 4200 ps.

dues in the range from $(\mathrm{P}-6)$ to $(\mathrm{P}-11)$. Kemptide does not possess residue beyond $\mathrm{P}-4$, hence it should not be interfered with by this structural change of the peptide binding site.

Similarly, the regulatory-subunit (RI $\alpha$ ) of cAMPdependent protein kinase should have reduced affinity for the E230Q mutant $\mathrm{C}$-subunit due to the loss of P-2 Arg:Glu230 electrostatic interaction. However, the C-subunit has a specific high-affinity binding domain for RI $\alpha$ that differs from that for $\mathrm{PKI} ;{ }^{34}$ the E230Q mutation may have limited effect on RI $\alpha$. The pseduosubstrate section of RI $\alpha$ does not pack closely against the Phe238-to-Gln242 section of the F-to-G helix loop; the binding of RI $\alpha$ may not be affected by the F-to-G helix loop conformational change that appears in the E230Q mutant.

\section{CONCLUSION}

In this study, we performed 10,000-ps MD simulations and automated docking simulations for the wild-type and the E230Q mutant C-subunits. The docking results suggest that hinge-bending fluctuation of the open conformation may not have much influence on PKI binding to both the wild-type and the E230Q mutant.
Instead, we found that the width of the peptide binding site, which was measured by the distance between Glu170 and Asp241, appears to regulate PKI binding. The results suggest that the F-to-G helix loop of the enzyme, together with the Asp241 in this loop section, might play a crucial role in the loss of E230Q mutant binding affinity for PKI. The conformation of this loop may be maintained by the long-range electrostatic interactions among Asp241 and the other acidic residues (Glu170, Glu203 and Glu230) in the peptide binding site. The Glu230-to-Gln mutation of the enzyme weakens the negative electrostatic potential in the peptide binding site and causes substantial loss of electrostatic repulsion among these acidic residues. In order to return to an energetic balance between the electrostatic repulsion and the other interactions, the F-to-G helix loop may adopt an altered conformation in order to achieve this balance. However, the altered conformation may hinder PKI binding, causing the E230Q mutant to fail to bind PKI properly.

This work was supported in part by the NIH, NSF, the Howard Hughes Medical Institute, National Biomedical Computing Resource, the NSF Center for Theoretical Biological Physics, SDSC, the W.M. Keck Foundation, and Accelrys, Inc. We thank Drs. Jian Wu and Jie Yang for helpful discussions. 


\section{REFERENCES}

1. Taylor, S. S.; Buechler, J. A.; Yonemoto, W. Annu Rev Biochem 1990, 59, 971-1005.

2. Knighton, D. R.; Zheng, J. H.; Ten Eyck, L. F.; Ashford, V. A.; Xuong, N.-H.; Taylor, S. S.; Sowadski, J. M. Science 1991, 253, 407-414.

3. Taylor, S. S.; Knighton, D. R.; Zheng, J. H.; Ten Eyck, L. F.; Sowadski, J. M. Annu Rev Cell Biol 1992, 8, 429-462.

4. Kemp, B. E.; Graves, D. J.; Benjamini, E.; Krebs, E. G. J Biol Chem 1977, 252, 4888-4894.

5. Gibbs, C. S.; Zoller, M. J. J Biol Chem 1991, 266, 8923-8931.

6. Hanks, S. K.; Quinn, A. M.; Hunter, T. Science 1988, $241,42-52$.

7. Tsigelny, I.; Grant, B. D.; Taylor, S. S.; Ten Eyck, L. F. Biopolymers 1996, 39, 353-365.

8. Grant, B. D.; Tsigelny, I.; Adams, J. A.; Taylor, S. S. Protein Sci 1996, 5, 1316-1324.

9. Wu, J.; Yang, J.; Kannan, N.; Madhusudan; Xuong, N.-H.; Ten Eyck, L. F.; Taylor, S. S. Protein Sci 2005, 14, 2871-2879.

10. Yang, J.; Garrod, S. M.; Deal, M. S.; Anand, G. S.; Woods, V. L.; Taylor, S. S. J Mol Biol 2005, 346, 191-201.

11. Lin, J.-H.; Perryman, A. L.; Schames, J. R.; McCammon, J. A. J Am Chem Soc 2002, 124, 5632-5633.

12. Zheng, J. H.; Trafny, E. A.; Knighton, D. R.; Xuong, N.-H.; Taylor, S. S.; Ten Eyck, L. F.; Sowadski, J. M. Acta Crystallogr 1993, 49, 362-365.

13. Frisch, M. J. GAUSSIAN98 Revision A. 10, Gaussian, Inc., Pittsburgh, PA, 2001.

14. Cornell, W. D.; Cieplak, P.; Bayly, C. I.; Gould, I. R.; Merz, K. M.; Ferguson, D. M.; Spellmeyer, D. C.; Fox, T.; Caldwell, J. W.; Kollman, P. A. J Am Chem Soc 1995, 117, 5179-5197.

15. Darden, T.; York, D.; Pederson, L. J Chem Phys 1993, 98, 10089-10092.

16. Ryckaert, J. P.; Ciccotti, G.; Berendsen, H. J. C. J Comput Phys 1977, 23, 327-341.

17. Shaltiel, S.; Cox, S.; Taylor, S. S. Proc Natl Acad Sci U S A 1998, 95, 484-491.
18. Narayana, N.; Cox, S.; Xuong, N.-H.; Ten Eyck, L. F.; Taylor, S. S. Structure 1997, 5, 921-935.

19. Morris, G. M.; Goodsell, D. S.; Halliday, R. S.; Huey, R.; Hart, W. E.; Belew, R. K.; Olson, A. J. J Comput Chem 1998, 19, 1639-1662.

20. Morris, G. M.; Goodsell, D. S.; Huey, R.; Olson, A. J. J Comput-Aided Mol Des 1996, 10, 293-304.

21. Hauer, J. A.; Taylor, S. S.; Johnson, D. A. Biochemistry 1999, 38, 6774-6780.

22. Rao, M. S.; Olson, A. J. Proteins 1999, 34, 173-183.

23. Laederach, A.; Dowd, M. K.; Coutinho, P. M.; Reilly, P. J. Proteins 1999, 37, 166-175.

24. Nacro, K.; Bienfait, B.; Lee, J.; Han, K. C.; Kang, J. H.; Benzaria, S.; Lewin, N. E.; Bhattacharyya, D. K.; Blumberg, P. M.; Marquez, V. E. J Med Chem 2000, 43, 921-944.

25. Rosenfeld, R. J.; Goodsell, D. S.; Musah, R. A.; Morris, G. M.; Goodin, D. B.; Olson, A. J. J Comput-Aided Mol Des 2003, 17, 525-536.

26. Baker, N. A.; Sept, D.; Joseph, S.; Holst, M. J.; McCammon, J. A. Proc Natl Acad Sci U S A 2001, 98, 10037-10041.

27. Cheng, X. D.; Shaltiel, S.; Taylor, S. S. Biochemistry 1998, 37, 14005-14013.

28. Lu, B. Z.; Wong, C. F.; McCammon, J. A. Protein Sci 2005, 14, 159-168.

29. Tsigelny, I.; Greenberg, J. P.; Nichols, W. L.; Taylor S. S.; Ten Eyck L. F. Biopolymers 1999, 50, 513-524.

30. Narayana, N.; Cox, S.; Shaltiel, S.; Taylor, S. S.; Xuong, N.-H. Biochemistry 1997, 36, 4438-4448.

31. Batkin, M.; Schvartz, I.; Shaltiel, S. Biochemistry 2000, 39, 5366-5373.

32. Karlsson, R.; Zheng, J. H.; Xuong, N.-H.; Taylor, S. S.; Sowadski, J. M. Acta Crystallogr 1993, 49, 381-388.

33. Johnson, D. A.; Akamine, P.; Radzio-Andzelm, E.; Madhusudan; Taylor, S. S. Chem Rev 2001, 101, 2243-2270.

34. Jim, C.; Xuong, N.-H.; Taylor, S. S. Science 2005, 307, 690-696.

Reviewing Editor: David Case 